\title{
Mathematical Model of Airflow in the Lungs of Children I: Effects of Tumor Sizes and Locations
}

\author{
R. A. SEGAL ${ }^{a, *}, X \cdot$ GUAN $^{b}, M \cdot$ SHEARER $^{a}$ and T. B. MARTONEN ${ }^{c, \dagger}$ \\ ${ }^{a}$ Department of Mathematics, North Carolina State University, Raleigh, NC 27695; ${ }^{\mathrm{b}}$ Department of Medicine, Duke University, \\ Durham, NC 27710; 'National Health and Environmental Effects Research Laboratory, U.S. EPA, Mail Drop 74, RTP, NC 27711, and \\ Division of Pulmonary Diseases, Department of Medicine, University of North Carolina, Chapel Hill, NC 27599
}

(Received In final form 9 September [999)

\begin{abstract}
To contribute to the development of more effective aerosol therapy protocols in pediatric medicine, we examined airflow patterns in the lung of a four-year-old child. In particular, we addressed how the presence of tumors in airways affected the character of airflow patterns. To study the effects of tumors we employed a computational fluid dynamics package, FIDAP, to define flow conditions within a model lung. The results indicated that tumors have a pronounced affect on both (i) localized velocity profiles in airways and (ii) bulk flow distribution within the lung. By identifying the effects of physical factors on flow conditions the findings will lead to improved drug delivery regimens.
\end{abstract}

Keywords: Pediatric medicine, mathematical model, fluid dynamics, aerosol therapy, tumors

\section{INTRODUCTION}

A recent review of pediatric chest tumor literature documents the formation of tumors in the lungs of children (Meyer and Nicotra, 1998). Lung tumors have been reported in children as young as one month in age (Hancock et al., 1993). While nearly 400 childhood lung tumor case histories have been reported in the literature, Meyer and Nicotra (1998) suggest that pediatric lung tumors may be under reported.

Of the tumors reported, $24 \%$ were benign (Meyer and Nicotra, 1998). Benign tumors (e.g. papillomas and plasma cell granulomas) were located in the larynx, trachea and main bonchi, although papillomas have been found further in the lung, usually the result of spread due to resection (Kramer et al., 1985). Some work has been done to investigate the use of aerosol therapy for the treatment of papillomas (Morrison et al., 1993).

Malignant tumors (e.g. bronchogenic carcinomas and bronchial "adenomas") make up the majority of reported cases. The most common therapy for these cancers is surgical removal. While keeping the patient alive is of foremost importance, the patient's quality of life is also a consideration. Since lung resection leads to reduced pulmonary function, it would be beneficial to have another option for treating lung cancer. We suggest that aerosol chemotherapy is

\footnotetext{
*Supported by a CRAY grant through the North Carolina Supercomputing Center and by the ARO.

${ }^{\dagger}$ Corresponding Author: Tel: 001919541 7875; Fax: 001919541 4284; E-mail: martonen.ted@epamail.epa.gov
} 
a potential alternative to surgery (Tatsumura et al., 1993).

We would like to promote the use of targeted drug delivery to the tumor site wth the intent of enhancing the efficacy of inhaled pharmocologic drugs. Therefore, we have investigated how airflow patterns in the lungs of children are affected by tumors. Since pharmacologic particles are transported by air, the very presence of tumors could influence the delivery of drugs to them. To perform simulations we employed a computational fluid dynamics (CFD) software package, FIDAP (1993). The CFD code has proved to be a valuable tool in the medical laboratory; in previous investigations we have employed it to study the effects of naturally occurring airway features like cartilaginous rings (Martonen et al., 1994a) and carinal ridges (Martonen et al., 1994b) on airstream flow patterns.
The objective of our current investigation was two-fold. First of all, to ascertain how different tumor sizes and locations affect velocity fields in airways. Secondly, to examine how ventilatory parameters (including mechanical ventilation) affect airflow patterns around prescribed tumor orientations. The details of the latter study are presented in Part II. The overall intent of our research was to provide a scientific basis for more effective aerosol therapy protocols via targeted drug delivery.

\section{METHODS}

\section{Lung Morphology}

A model of the upper airways of the tracheobronchial tree within a child's lung is presented in Figure 1. It

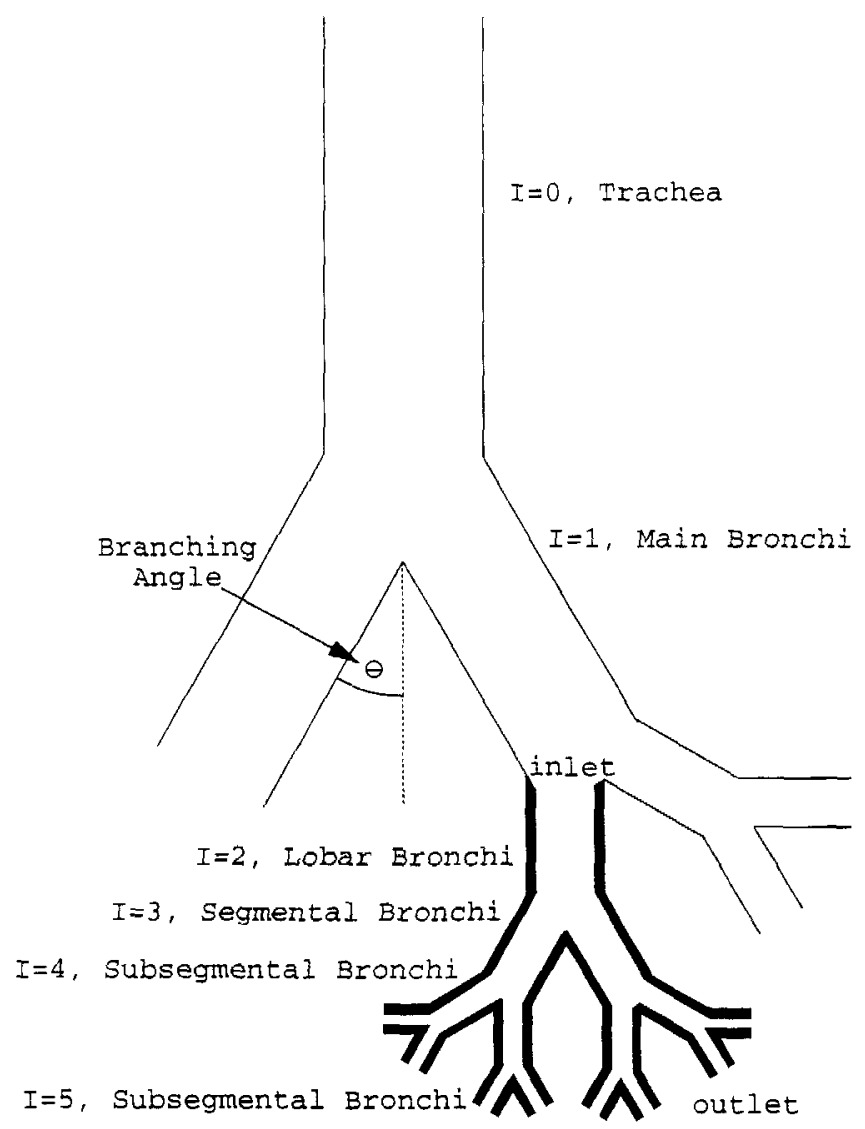

FIGURE 1 Lung morphology for airway generations $0 \leq \mathrm{I} \leq 5$. The heavy-lined region corresponds to the section of the tracheobronchial tree used in the simulations. 
is based on the symmetric, dichotomously branching network proposed by Weibel (1963) for the adult. We integrated that information with the findings of Cleveland (1979) and Hofmann et al. (1989) to define a child's lung. For children without an aortic indentation, Cleveland determined that the main bronchial branching angles were symmetric, with the left and right branching angles being approximately $30^{\circ}$ (see Figure 1). This result was also used to describe the other bifurcations. The dimensions of airways (diameters and lengths) for the lung of a four-year-old child were calculated using the formulas described by Hofmann et al. (1989) (see Table I). Each generation, I, contained $2^{\mathrm{I}}$ number of airways.

We focused on generations $I=2-5$ which corresponded to the lobar, segmental and two levels of subsegmental bronchi, respectively. We have chosen these airways for the following reasons.

1. We note that although many manuscripts in the literature discuss the treatment and general locations of carcinomas, specific data detailing their sites of origination are scarce. There is evidence suggesting that these malignant tumors can be found in the upper airways (Androssy et al., 1977; Augustin et al., 1987; Bellah et al., 1992; dePareder et al., 1970; Fontenelle, 1976; Hancock et al., 1993; Lack et al., 1983; Roberts, 1954; Verska and Conolly, 1986; Wilkins et al., 1963).

2. This work could potentially be used for the treatment of papillomas. There is evidence that these tumors may be located in the upper airways described by the model (Clements and Gavelle, 1986; Cohen et al., 1980; Kirchner,
1951; Kramer et al., 1985; Oleszczuk-Raszke and Cremin, 1988).

3. The motion of air in the lung is very complex. For instance, airflow in the trachea is dominated by the laryngeal jet, whose effects are propagated downstream (Martonen et al., 1993). It should be noted that the above work was done for the adult lung. There is no comparable work detailing such effects in a child's lung. However, the work of Martonen et al. (1989) indicates that the lung is not fully developed until well passed the age in question (age 4). We hypothesize that the effects of morphological features would be reduced in a child's lung.

\section{Tumors}

We performed an extensive and systematic series of theoretical simulations examining the effects of the following factors on airstream patterns in bronchial airways:

- sizes of individual tumors;

- positions of the tumors; and,

- hydrodynamic interactions between tumors.

We addressed two distinct aspects of the fluid dynamics patterns: (i) velocity fields within individual airways (i.e., localized effects); and, (ii) distribution of flow within the lung (i.e., bulk effects).

For brevity, only a few cases of the numerous simulations we have completed were selected for presentation. These examples document that tumors had pronounced effects on local velocity fields and will, therefore, affect the targeted delivery of inhaled

TABLE I Airway dimensions and flow parameters for a child's (age 4 years) lung and sedentary breathing.

\begin{tabular}{ccccc}
\hline Generation Number, I & $\begin{array}{c}\text { Diameter, D } \\
(\mathrm{cm})\end{array}$ & $\begin{array}{c}\text { Length, L } \\
(\mathrm{cm})\end{array}$ & $\begin{array}{c}\text { Mean Velocity, V } \\
(\mathrm{cm} / \mathrm{s})\end{array}$ & Re $^{*}$ \\
\hline 0 & 1.105 & 5.330 & 117 & 854 \\
1 & 0.804 & 2.540 & 110 & 584 \\
2 & 0.575 & 0.971 & 107 & 410 \\
3 & 0.436 & 0.673 & 94 & 270 \\
4 & 0.276 & 0.559 & 117 & 213 \\
5 & 0.216 & 0.455 & 95 & 137 \\
\hline
\end{tabular}

${ }^{\times} \operatorname{Re}=$ Reynolds number $=\mathrm{DV} / \nu$, where $\nu=$ kinematic viscosity of air 
drugs because particles are entrained and transported by airstreams. Moreover, when sufficiently developed in size $(r / D=0.4$, where $r$ is the tumor radius and $D$ is the airway diameter), the presence of the tumors actually caused the maldistribution of air within the lung.

The sizes of the tumors were related to the sizes of airways in which they were located by the ratio $r / D$. There were certain obvious constraints: for example, the radius of a tumor could not exceed the diameter of the airway in which it was located (in such a case How to downstream regions would be prohibited). A variety of sizes were used to cover different stages of tumor development. In Table $\mathrm{II}$, representative sizes for tumors located in generation $I=3$ airways are presented and their sizes are given as a percentage of the airway diameter.

A variety of different tumor locations were simulated (see Figure 2); namely, tumors were placed
TABLE II Tumor sizes in host airways

\begin{tabular}{cc}
\hline $\begin{array}{c}\text { Tumor Size, } r \\
(\mathrm{~cm})\end{array}$ & $\begin{array}{c}\text { Percentage of Airway Diameter } \\
\left.(\mathrm{r} / \mathrm{D})^{*} 100\right)\end{array}$ \\
\hline 0.087 & $20 \%$ \\
0.131 & $30 \%$ \\
0.174 & $40 \%$ \\
\hline
\end{tabular}

on the carinal ridge and on the side walls of an airway. We have performed independent fiberoptic bronchoscopy examinations of human subjects to study morphological features of carinal ridges and airway walls (Martonen et al., 1994a,b). There is clinical evidence that these are appropriate choices for tumor placement (Oho and Amemiya, 1980) (see Figures 3 and 4). For tumors placed on the walls, a model constraint was that the initial point of contact remained constant; that is, the tumor began $0.0673 \mathrm{~cm}$ from the bifurcation point (corresponding

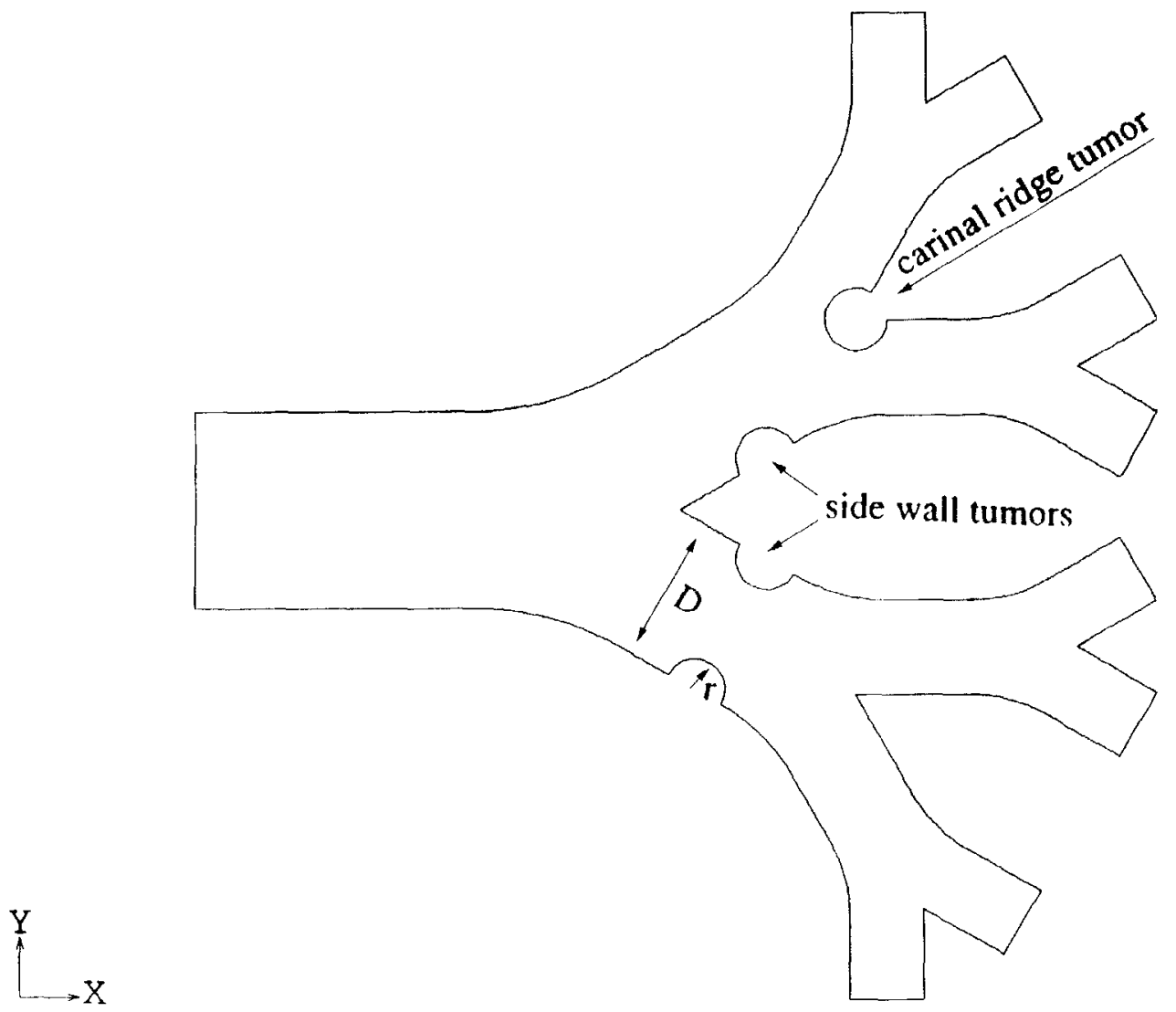

FIGURE 2 Prescribed Positions of Tumors in the Airway Network. 


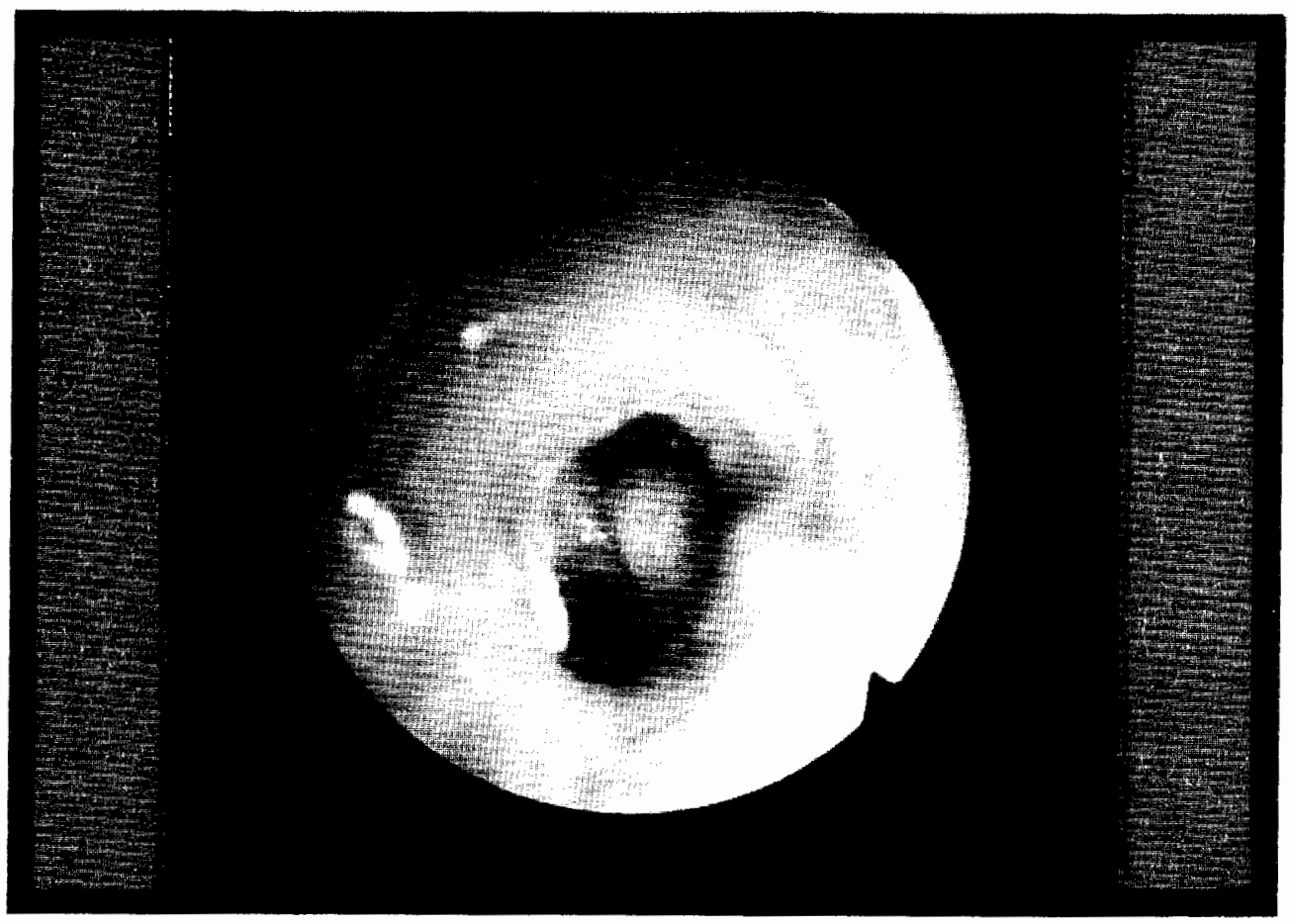

FIGURE 3 Photograph of a tumor located on a carinal ridge taken during fiberoptic bronchoscopy. Used with permission (Oho and Amemiya, 1980) (sce colour plate I).

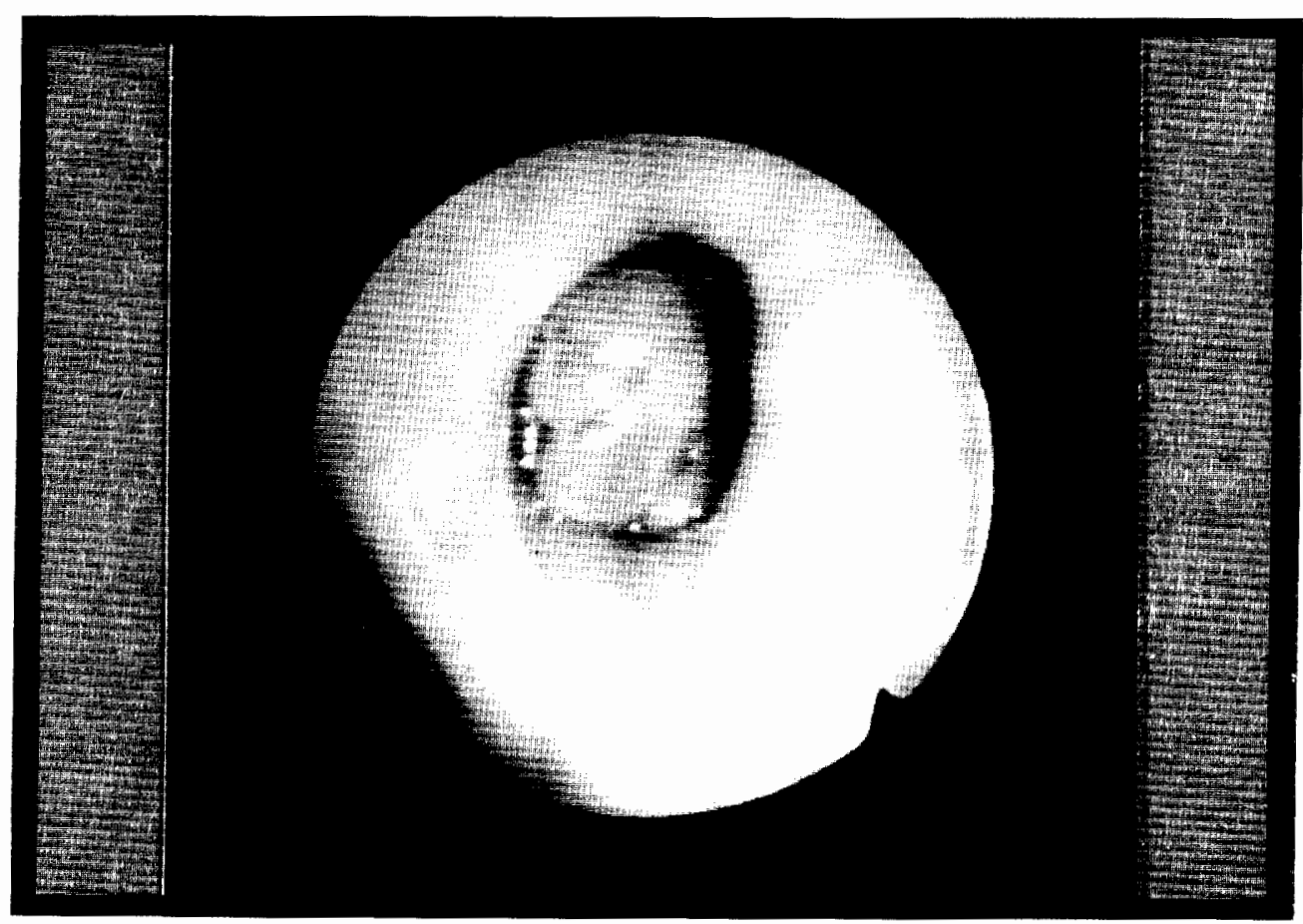

FIGURE 4 Photograph of a tumor located on a side wall of the lung taken during fiberoptic bronchoscopy. Used with permission (Oho and Amemiya, 1980) (see colour plate II). 
to $10 \%$ of the length of the generation $\mathrm{I}=3$ airway in which the tumor was located) regardless of the size of the tumor.

\section{Velocity Profiles}

A sedentary condition was assumed in this study (i.e., Part I). The corresponding tidal volume and breathing frequency are $V_{T}=152 \mathrm{ml}$ and $f=$ 22 breaths/min, respectively. This breathing pattern was consistent with the goal of providing more effective aerosol therapy to resting patients. The volumetric flow rate entering the trachea during inspiration for a four-year-old child is $112 \mathrm{~cm}^{3} / \mathrm{s}$, obtained by dividing $V_{T}$ by the inspiratory phase time $(60 / 2 f)$ of a breath (Martonen et al., 1989). The corresponding volumetric flow rate for one airway in generation $\mathrm{I}=2$ is $28 \mathrm{~cm}^{3} / \mathrm{s}$, obtained by dividing the total flow rate entering the lung by 4 (the number of airways in generation $I=$ 2 ). The mean velocity of the air as it enters a generation $\mathrm{I}=2$ airway is determined by dividing the volumetric flow rate by the airway's crosssectional area $\left((0.2874)^{2} \pi \mathrm{cm}^{2}\right)$. The mean velocity is $107 \mathrm{~cm} / \mathrm{s}$. Flow parameters for the $0 \leq I \leq 5$ airways are presented in Table I. The Reynolds

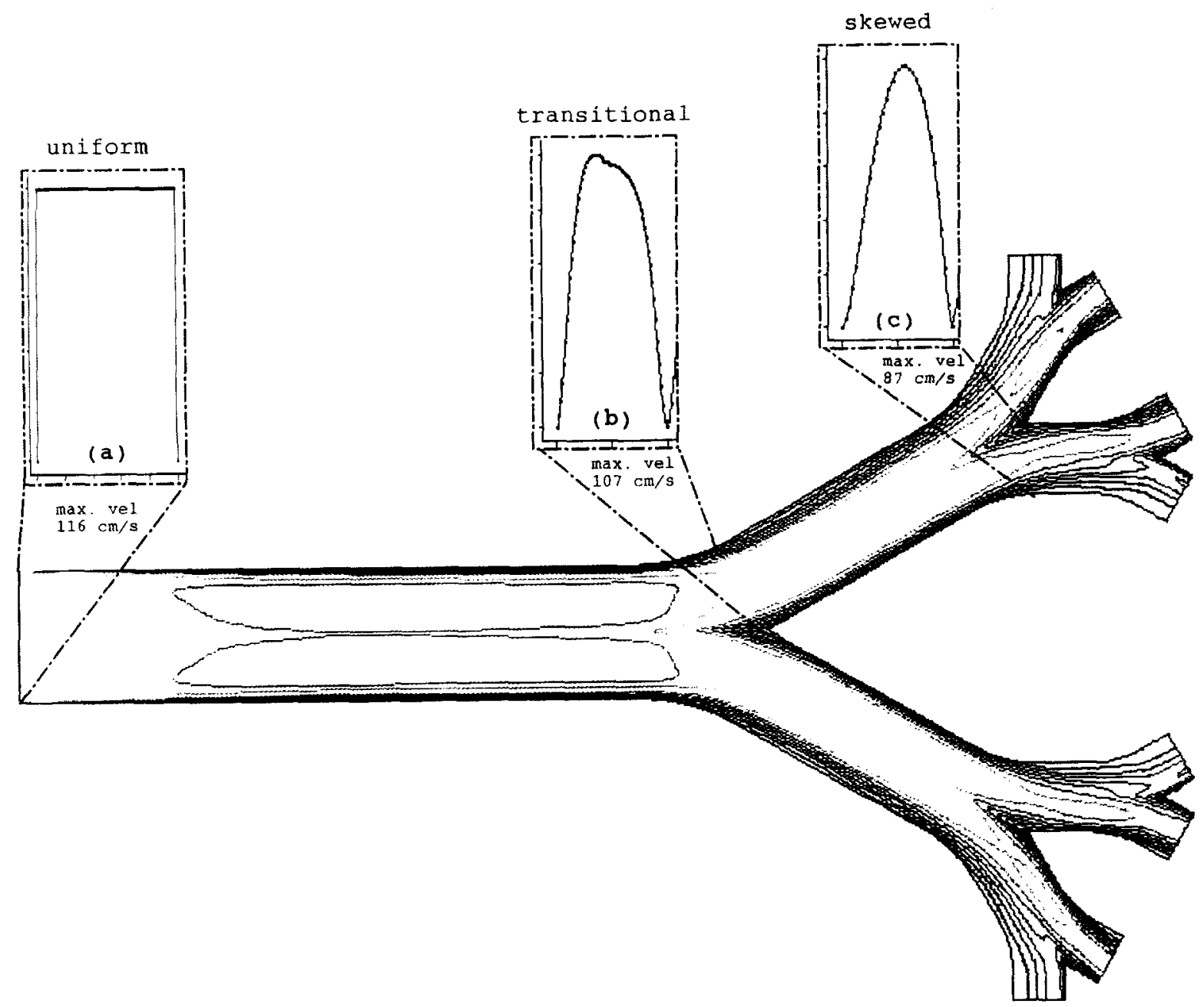

FIGURE 5 Computed velocity profiles at the entrance to each generation assuming a uniform inlet profile corresponding to a sedentary breathing rate. 
necessary to define the mesh (i.e., the grid configuration and the element size) on which the solution was based. An example of the mesh used in the simulations is shown in Figure 6, where the airways are labeled according to the following convention: $\mathrm{I}^{n}$, where I indicates the generation number and $n$ distinguishes the airways within each generation. This system will be used to refer to specific airways in the following Results section.

\section{RESULTS}

We focused on effects of (1) tumor size and (2) tumor placement on airflow patterns. A sedentary breathing condition was simulated which corresponded to an average velocity of $107 \mathrm{~cm} / \mathrm{s}$ at the inlet to airway generation $\mathrm{I}=2$.

\section{Comparison of Different Inlet Velocity Profiles}

To establish a control case, we began with an analysis of airflow with no tumors present in the airways. Three different inlet velocity profiles were considered: uniform (see Figure 7), parabolic (see Figure 8), and skewed (see Figure 9). All three inlet profiles created a downstream flow pattern which was weighted along the inner wall distal to the first bifurcation. Of the three cases, the uniform inlet profile produced the largest region of core flow and consequently had the smallest region of reduced flow along the bounding walls. [Note: The core flows are the red/orange/yellow regions which begin at the entrance to generation $I=2$ in the illustrations. They are the regions of highest velocities.] The uniform inlet velocity profile case did not attain the relatively higher core flow velocity values experienced with

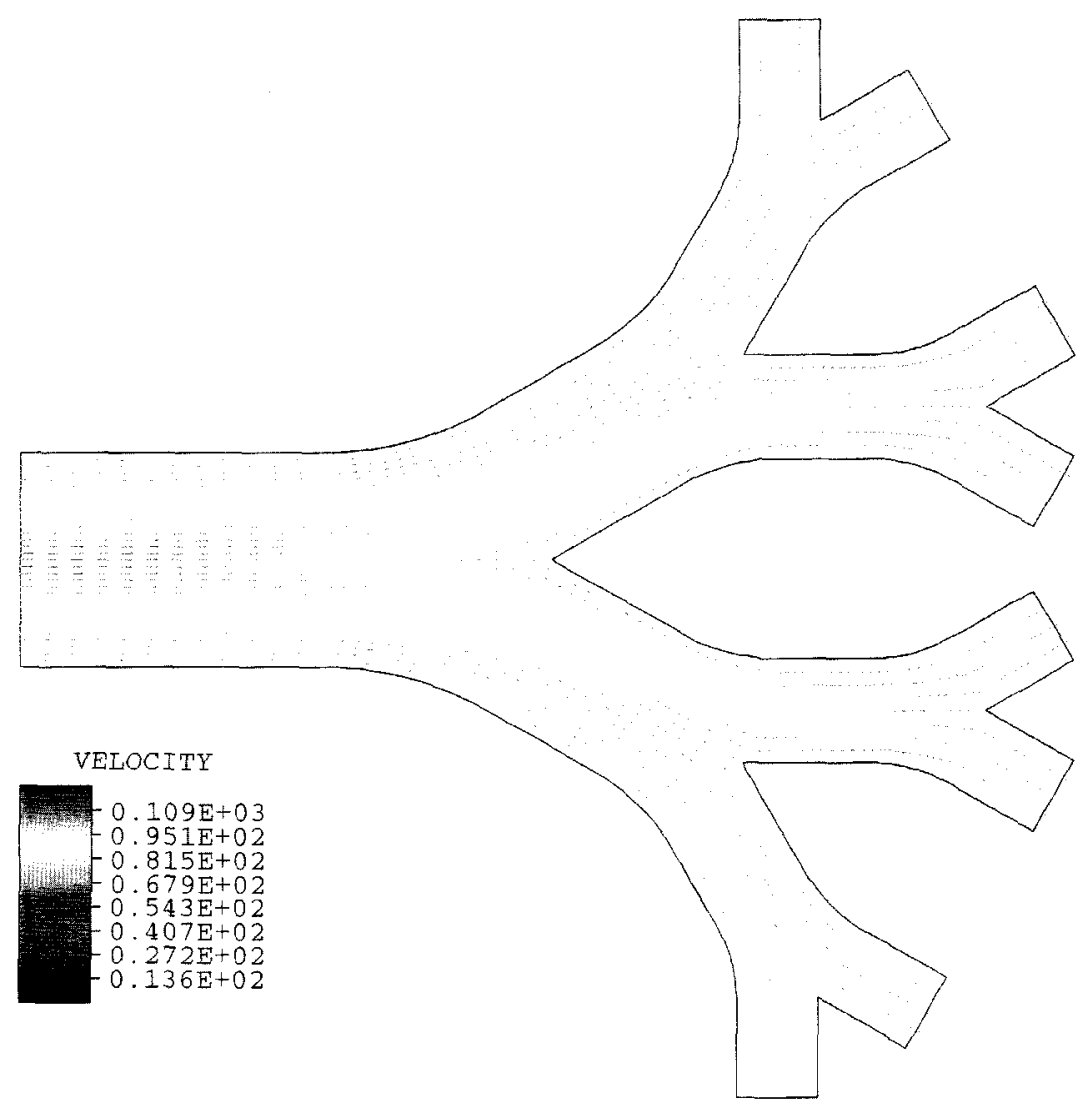

FIGURE 8 Velocity fields in generations $2 \leq \mathrm{I} \leq 5$ of a four-year-old's lung. This simulation assumes a parabolic inlet velocity profile corresponding to a sedentary breathing rate (see colour plate IV). 


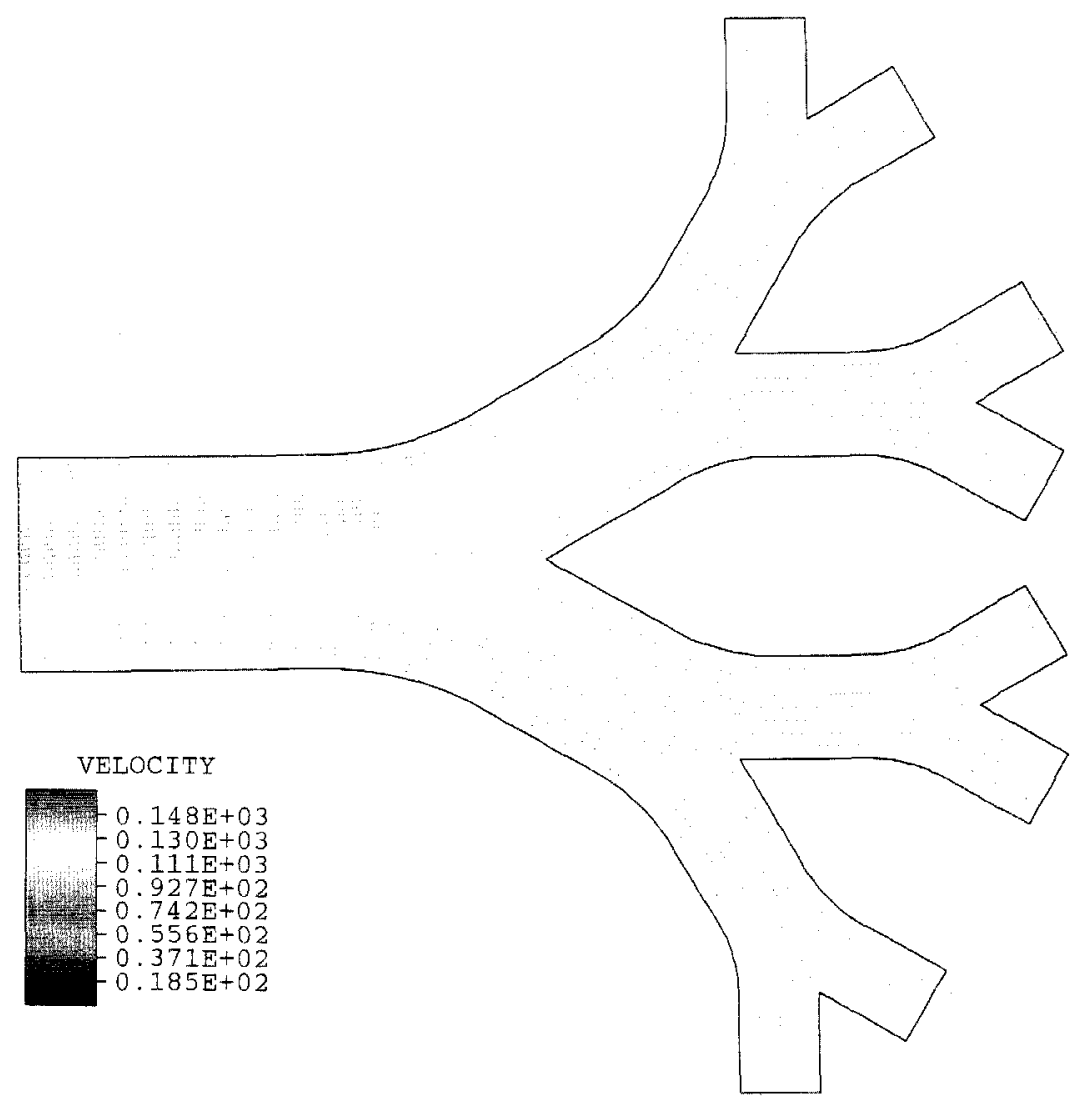

FIGURE 9 Velocity fields in generations $2 \leq \mathrm{I} \leq 5$ of a four-year-old's lung. This simulation assumes a skewed inlet velocity profile corresponding to a sedentary breathing rate (see colour plate $\mathrm{V}$ ).

the other two cases because the region of core flow was larger. The skewed profile and parabolic profile cases were similar to each other regarding the sizes of the respective core regions and the velocity values in them. This was not unexpected because the skewed profile had a quasi-parabolic shape. The skewed profile, however, was not symmetric and the volumetric flow rate in region $3^{b}$ was greater than that in region $3^{a}$.

Because the skewed profile entering $I=2$ was derived from simulating flow through the proximal (i.e., upstream) region of the lung, it was reasonable to consider this inlet profile to be a more accurate portrayal of real (i.e., in vivo) conditions than either of the symmetric (uniform or parabolic) profiles. Therefore, the skewed inlet profile was used in the simulations discussed below.

\section{Effects of an Isolated Tumor}

Let us first address localized effects. The findings indicate that tumor effects are first noticeable when $\mathrm{r} / \mathrm{D}=0.1$. The clinical significance of this observation is that even what may be considered to be small tumors affect localized flow fields.

Now, let us consider flow distribution within the lung (i.e., bulk effects). The presence of a single tumor in an airway caused a change in velocity patterns among airways; this is apparent following comparisons of Figure 9 (the control case), with Figures 10 and 11. For instance, regardless of the position of a tumor within region $3^{b}$, the flow rate in region $3^{a}$ was greater. Moreover, a strong separation of the core flow in upstream airway region 2 occurred well before the bifurcation to region 3 . The flow was directed to other regions of the lung, in 


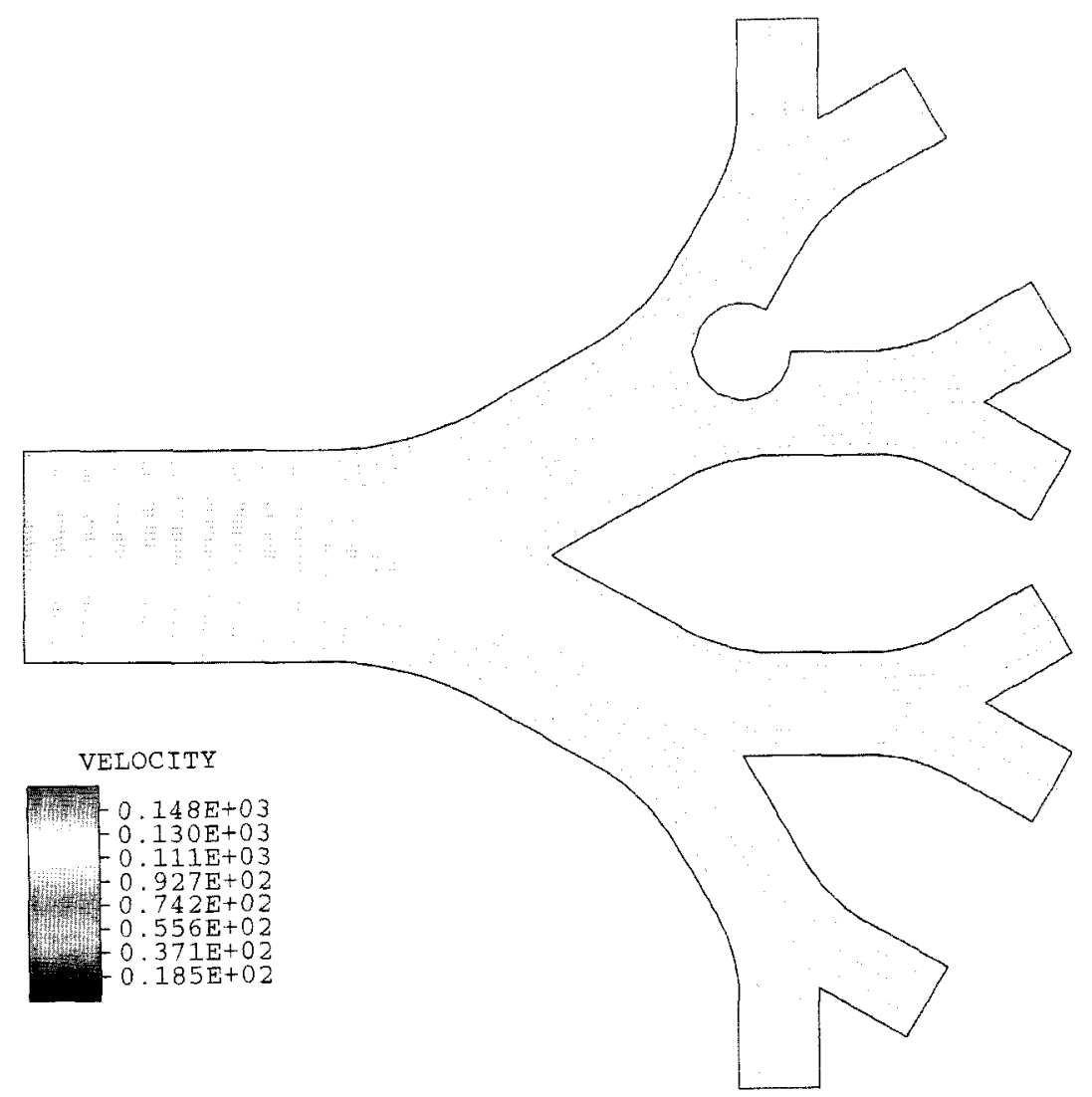

FIGURE 10 Velocity field in generations $2 \leq \mathrm{I} \leq 5$ of a four-year-old's lung with a skewed inlet velocity profile corresponding to a sedentary breathing rate. The tumor located on the carinal ridge has the radius $\mathrm{r}=0.131 \mathrm{~cm}$ (see colour plate VI).

this simulation, $4^{b}$ and $4^{c}$. The magnitude of tumorinduced effects were quite pronounced. The flow in the region distal to (i.e., directly behind) the tumor slowed to approximately one tenth of the value above the tumor.

In the two subsections that follow, we detail the effects of a tumor located on a carinal ridge and the effects of a tumor located on a side wall.

\section{Tumor on the carinal ridge}

The presence of a tumor on the carinal ridge in region $3^{b}$ caused the core airflow to shift primarily to region $3^{a}$ and to increase in width relative to the core airflow in the absence of a tumor (see Figures 10 and 9, respectively). The maximum velocity decreased from $107 \mathrm{~cm} / \mathrm{s}$ when no tumor was present to $95 \mathrm{~cm} / \mathrm{s}$ when there was a tumor present on the carinal ridge. As the tumor size increased, the flow was directed to region $3^{a}$ and the flow volume downstream from (i.e., distal to) the tumor decreased. When the tumor radius was $40 \%$ of the airway diameter, the flow in region $5^{b}$ was nearly zero (see Figure 12) indicating maldistribution of airflow within the lung.

\section{Tumor on the inside wall}

The occurrence of a tumor on the inside wall of region $3^{b}$ caused the core flow in region $3^{a}$ to increase in scope (physical size) and velocity magnitude relative to flow conditions when no tumors were present (see Figure 11 and 9, respectively). The maximum velocity in region 3 increased from $107 \mathrm{~cm} / \mathrm{s}$ to $125 \mathrm{~cm} / \mathrm{s}$. The air flow remaining in 


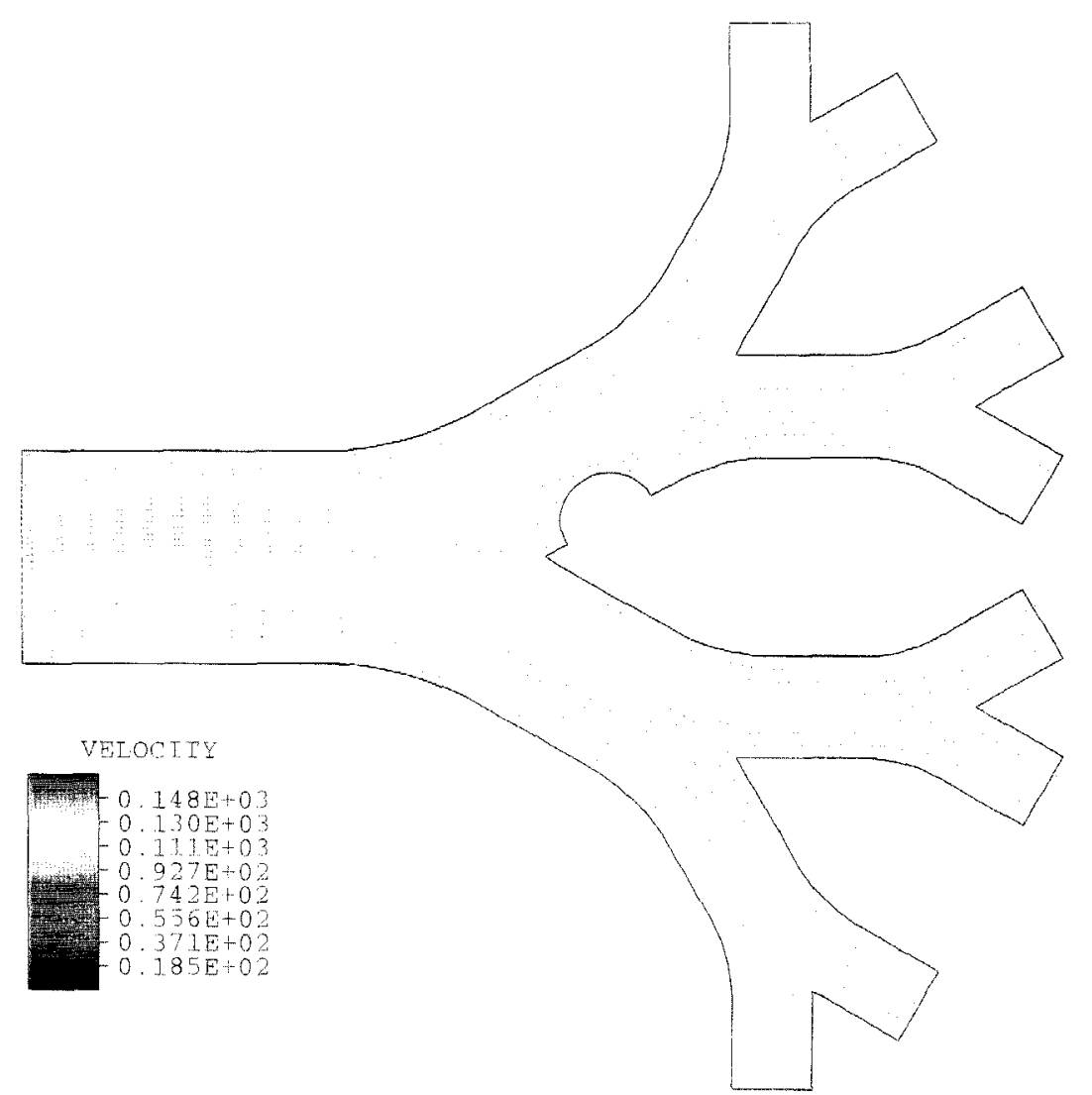

FIGURE 11 Velocity field in generations $2 \leq \mathrm{I} \leq 5$ of a four-year-old's lung with a skewed inlet velocity profile corresponding to a sedentary breathing rate. The tumor located on the inside wall has the radius $\mathrm{r}=0.131 \mathrm{~cm}$ and is located $0.0673 \mathrm{~cm}$ from the carinal ridge (see colour plate VII).

region $3^{b}$ became focused, creating a region of higher velocity. As the tumor size increased, this effect was enhanced.

\section{Interactive Effects}

Finally, we examined effects introduced by multiple tumors. In particular, we considered the influence a second tumor had on the flow around a tumor on the carinal ridge in region $3^{b}$. The carinal ridge tumor had a radius of $0.131 \mathrm{~cm}$. The second tumor was located on the inner wall and also had a radius of $0.131 \mathrm{~cm}$ (see Figure 13). Following a comparison of Figure 13 with Figures 10 and 11, we observed that the bulk flow patterns were nearly identical to the bulk flow patterns in Figure 10 where the only tumor present was located on the carinal ridge. Clearly, the carinal ridge tumor had the dominant effect (i.e., with respect to the second tumor) on the overall flow pattern.

\section{DISCUSSION}

To contribute to the development of more effective aerosol therapy protocols in pediatric medicine, we have quantified the effects of tumors on airflow patterns within airways. We studied both localized and bulk flow fields. Tumors located on carinal ridges exhibited the most pronounced effects on flow fields. If we increased the radius of a carinal ridge tumor to $40 \%$ of the airway diameter, a maldistribution of airflow occurred. 


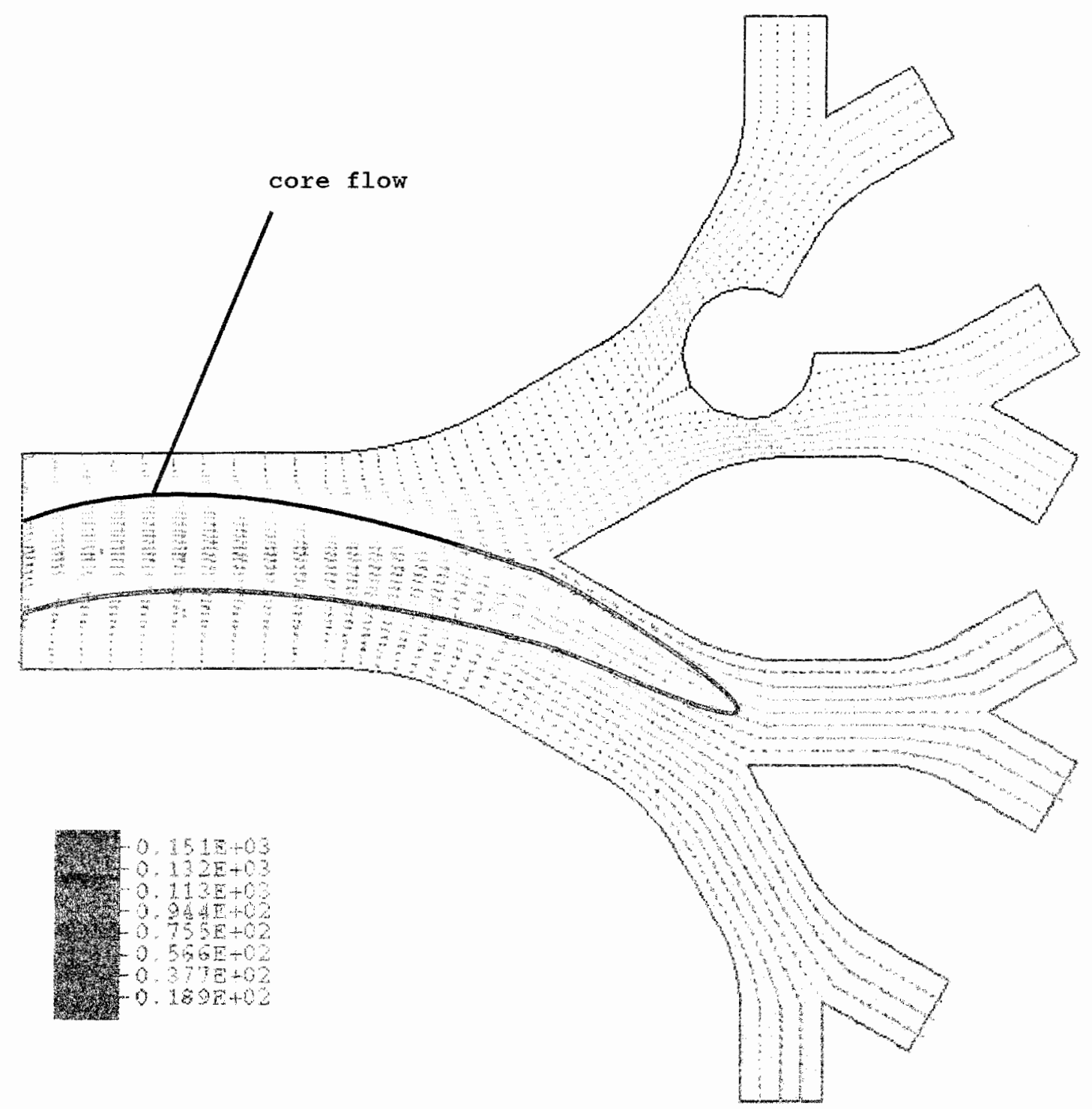

FIGURE 12 Velocity field in generations $2 \leq I \leq 5$ of a four-year-old's lung with a skewed inlet velocity profile corresponding to a sedentary breathing rate. The tumor located on the carinal ridge has the radius $\mathrm{r}=0.174 \mathrm{~cm}$ (see colour plate VIII).

The presence of a tumor on the inner wall caused a slight change in bulk flow patterns but these effects were small when compared to the aforementioned effects of carinal ridge tumors. In each case considered, the flow downstream of a tumor was approximately one tenth the magnitude of the flow upstream from it.

The choice of inlet velocity profiles had an effect on downstream flow patterns within bifurcations.
Because a skewed inlet velocity profile is the most physiologically realistic, it is to be preferred in theoretical simulations.

By identifying the effects of structural factors on flow conditions, we hope to promote the use of inhaled drugs in the treatment of airway tumors. Applications for such models may include aerosol therapy for other respiratory tract problems including viral disease (Knight and Gilbert, 1990), children 


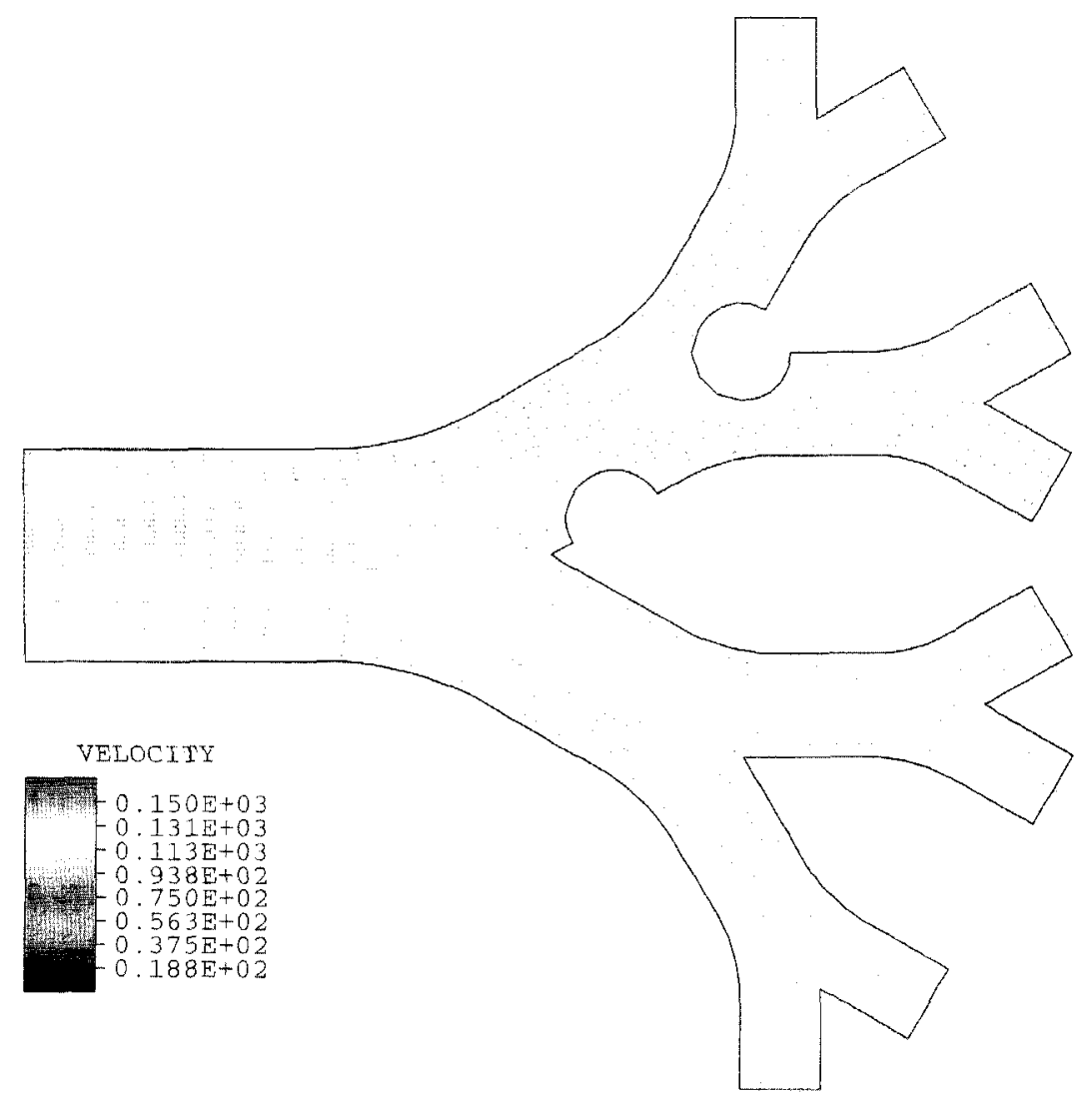

FIGURE 13 Velocity field in generations $2 \leq \mathrm{I} \leq 5$ of a four-year-old's lung with inlet velocity corresponding to a sedentary breathing rate. The tumor located on the carinal ridge has the radius $r=0.131 \mathrm{~cm}$; the tumor located on the inside wall the radius of $r=0.131 \mathrm{~cm}$ and is located $0.067 \mathrm{~cm}$ from the bifurcation spur (see colour plate IX).

with asthma (Simons, 1997), and the effects of lung occlusions on airflow patterns in the lungs of children with cystic fibrosis (Martonen et al., 1997).

\section{References}

[1] Andrassy, R. J., Feldtmann, R. W. and Stanford, W. (1977). Bronchial carcinoid tumors in children and adolescents. Joumal of Pediatric Surgery, 12, 513-517.

[2] Asgharian, B. and Anjilvel, S. (1994). Inertial and gravitational deposition of particles in a square cross section bifurcating airway. Aerosol Science and Technology, 20, $177-193$.

[3] Augustin, N., Hofmann-V. Kap-herr, S. and Wuring, P. (1987). Endotracheal and endobronchial tumors in childhood. Progress in Pediatric Surgery, 21, 136-144.

[4] Bellah, R. D., Mahboubi, S. and Berdon, W. E. (1992). Malignant endobronchial lesions of adolescence. Pediatric Radiology, 22, 563-567.

[5] Clements, R. and Gravelle, I. H. (1986). Laryngeal Papillomatosis. Clinical Radiology, 37, 547-550.
16] Cleveland. R. H. (1979). Symmetry of Bronchial Angles in Children. Pediatric Radiology, 133, 89-93.

[7] Cohen, S. R., Seltzer, S., Geller, K. A. and Thompson, J. W. (1980). Papilloma of the larynx and tracheobronchial tree in children. Annals of Otology. Rhinology, and Laryngology, 89, 497-503.

[8] dePareder, C. G., Pierce, W. S., Groff, D. B. and Waldhausen, J. A. (1970). Bronchogenic tumors in children. Archives of Surgery, 100, 574-576.

[9] FIDAP. (1993). Fluid Dynamics International. Evanston. Illinois.

[10] Fontenelle, L. J. (1976). Primary adenocarcinoma of lung in a child: a review of the literature. American Surgery, 42, 296.

[11] Hancock, B. J., Di Lorenzo, M., Youssef, S.. Yazbeck, S., Marcotte, J. E. and Collin, P. P. (1993). Childhood primary pulmonary neoplasms. Joumal of Pediatric Surgery, 28, $1133-1136$.

[12] Heistracher, T. and Hofmann, W. (1995). Physiologically realistic models of bronchial airway bifurcations. Journal of Aerosol Science, 26, 497-509.

[13] Hofmann, W., Martonen, T. B. and Graham, R. C. (1989). Predicted deposition of nonhygroscopic aerosols in the human lung as a function of subject age. Journal of Aerosol Medicine, 2, 49-68. 
[14] Kirchner, J. A. (1951). Papilloma of the larynx with extensive lung involvement. Laryngoscope, 61, 1022-1029.

[15] Knight, V. and Gilbert, B. E. (1990). Aerosol treatment of respiratory viral disease. Lung, Suppl, 406-413.

[16] Kramer. S. S., Wehunt, W. D., Stocker, J. T. and Kashima, H. (1985). Pulmonary Manifestations of Juvenile Laryngotracheal Papillomatosis. AJR, 144, 687-694.

[17] Lack, E. E., Harris, G. B. C., Eraklis, A. J. and Vawter, G. F. (1983). Primary bronchial tumors in childhood: A clinicopathologic study of six cases. Cancer, 51, 492-497.

[18] Martonen, T. B., Graham, R. C. and Hoffman, W. (1989) Human subject age and activity level: Factors addressed in a biomathematical deposition program for extrapolation modeling. Health Physics. 57, 49-59.

[19] Martonen, T. B.. Zhang, Z. and Lessmann, R. (1993). Fluid dynamics of the human larynx and upper tracheobronchial airways. Aerosol Science and Technology, 19, 133-156.

[20] Martonen, T. B., Yang, Y. and Xue, Z. Q. (1994a). Influences of cartilaginous rings of tracheobronchial fluid dynamics. Inhalation Toxicology, 6, 185-203

[21] Martonen, T. B., Yang, Y. and Xue, Z. Q. (1994b). Effects of carinal ridge shapes on lung airstreams. Aerosol Science and Technology, 21, 119-136.

[22] Martonen. T. B., Hwang, D., Katz, I., Yang, Y. and Guan, X. (1997). Cystic fibrosis: treatment with a supercomputer drug delivery model. Advances in Engineering Software, 28, 359-364.

[23] Meyer. J. S. and Nicotra, J. J. (1998). Tumors of the pediatric chest. Seminars in Roentgenology, 33, 187-198.

[24] Morrison, G. A. J., Kotecha, B. and Evans, J. N. G. (1993). Ribavirin treatment for juvenile respiratory papillomatosis The Journal of Laryngology and Otology, 107, 423-426.
[25] Oho, K. and Amemiya, R. (1980). Practical Fiberoptic Bronchoscopy. IGAKU-SHOIN Ltd., Tokyo, Japan.

[26] Oleszczuk-Raszke, K. and Cremin, B. J. (1988). Computed tomography in pulmonary papillomatosis. The British Journal of Radiology, 61, 160-161.

[27] Olson, D. E., Dart, G. A. and Filley, G. F. (1970). Pressure drop and fluid flow regime of air inspired into the human lung. Joumal of Applied Physiology, 28, 482-494.

[28] Roberts, K. D. (1954). Bronchial adenoma on childhood Archives of Diseases in Childhood, 29, 360-362.

[29] Schroter, R. C. and Sudlow, M. F. (1969). Flow patterns in models of the bronchial airways. Respiration physiology, 7. $341-355$.

[30] Simons, F. E. R. (1997). A comparison of Beclomethasone, Salmeterol and placebo in children with asthma. The New England Journal of Medicine, 337, 1659-1665.

[31] Tatsumura, T., Koyama, S., Tsujimoto, M., Kitagawa, M. and Kagamimori, S. (1993). Further study of nebulisation chemotherapy, a new chemotherapeutic method in the treatment of lung carcinomas: fundamental and clinical. British Journal of Cancer, 68, 1146-1149.

[32] Verska, J. J. and Connolly, J. E. (1986). Bronchial adenomas in children. Journal of Thoracic Cardiovascular Surgery, 55, 411-417.

[33] Wilkins, E. W. Jr., Darting, R. C., Soutter, L. and Sniffen, R. C. (1963). A continuing clinical survey of adenomas of the trachea and bronchus in a general hospital. Journal of Thoracic Cardiovascular Surgery, 46, 279.

[34] Weibel, E. R. (1963). Morphometry of the human lung. Academic Press, New York. 


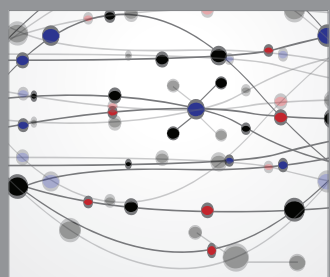

The Scientific World Journal
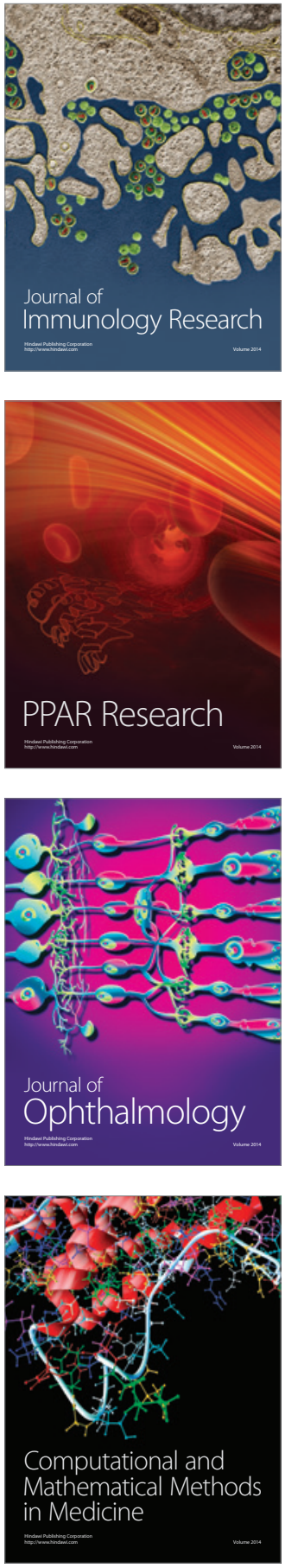

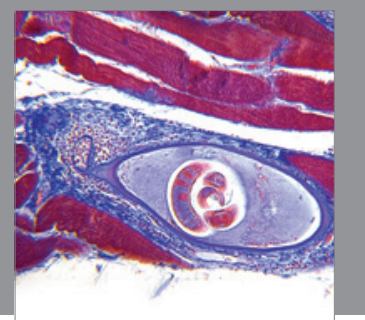

Gastroenterology

Research and Practice
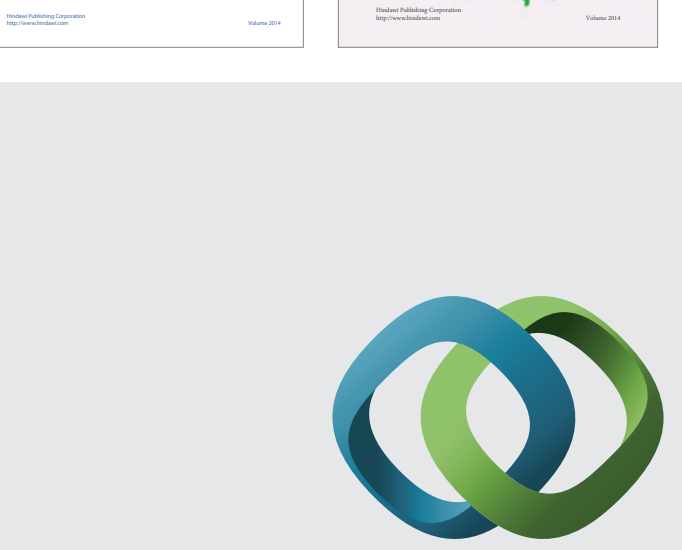

\section{Hindawi}

Submit your manuscripts at

http://www.hindawi.com
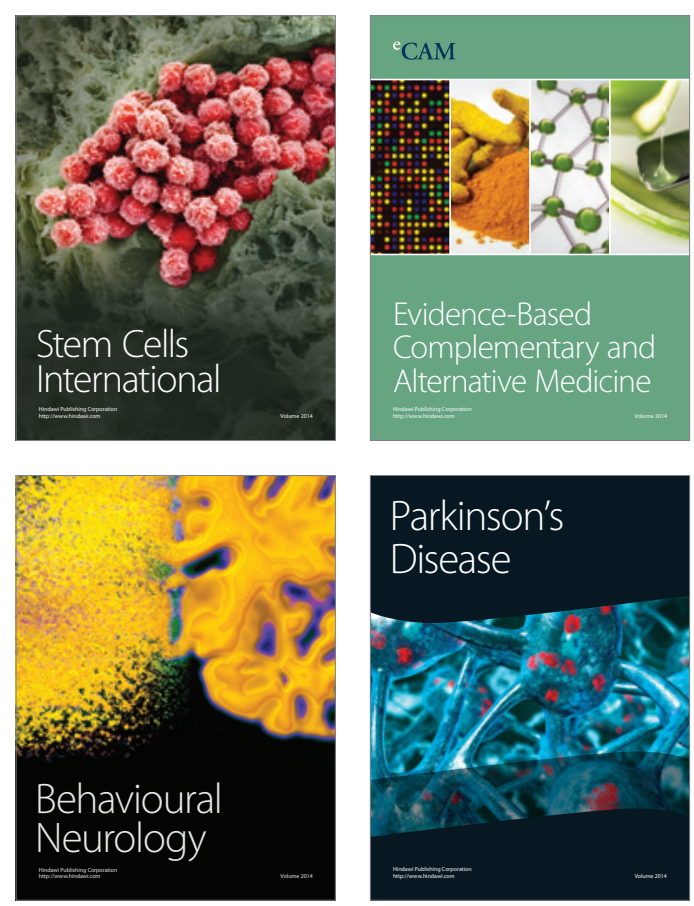

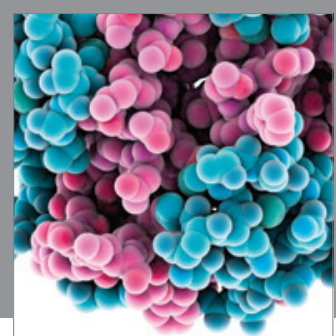

Journal of
Diabetes Research

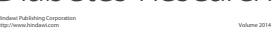

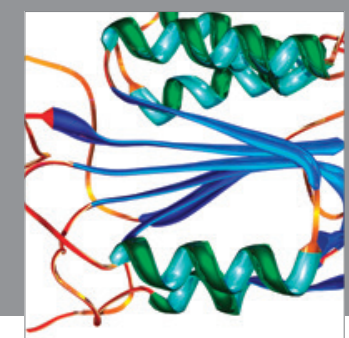

Disease Markers
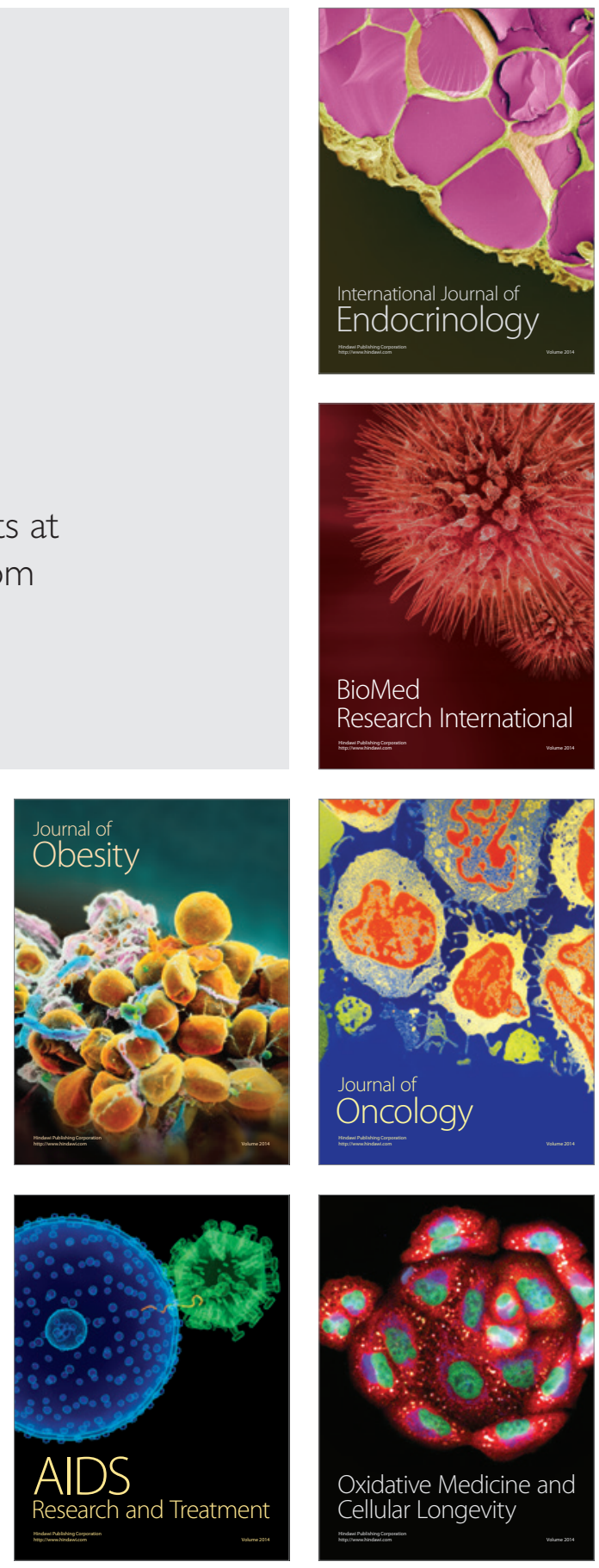